\title{
First Light for Station V of the Low-Frequency All-Sky Monitor Radio Telescope
}

\author{
Timothy Dolch*, Philip Andrews*, Brent Cole ${ }^{\dagger}$, Teviet Creighton ${ }^{\dagger}$, Louis P. Dartez ${ }^{\dagger}$, \\ Alexander Dulemba*, Fredrick A. Jenet ${ }^{\dagger}$, Sashabaw Niedbalski*, Caleb Ramette*, Shane Smith* \\ ${ }^{*}$ Department of Physics, Hillsdale College, Hillsdale, MI, USA, Email: tdolch@ @illsdale.edu \\ $\dagger$ Center for Advanced Radio Astronomy, University of Texas Rio Grande Valley, Brownsville, TX, USA
}

\begin{abstract}
The Low-Frequency All-Sky Monitor is a radio telescope network consisting of five stations, each with 13 LongWavelength Array (LWA) antennas, distributed around the North American continent. The LWA antennas are arranged so as to form a wide-angle drift scan beam, sensitive to bright radio transients, astrophysical and ionospheric, at 10-88 MHz. The multiple stations can reliably verify astrophysical events by simultaneous detection. Adding the fifth station at Hillsdale College in Hillsdale, MI, provides a wider range of declination coverage for the project. Station $\mathrm{V}$, like the previous four stations, was constructed by undergraduate researchers, who continue to operate and manage the telescope. Here we report on Station V's "first light", and discuss future prospects.
\end{abstract}

\section{INTRODUCTION}

The Low-Frequency All-Sky Monitor (LoFASM) is a distributed array of LWA antenna clusters. Each has a wide beam, but neither the individual stations nor LoFASM as a whole is intended to localize point source transients. By continuously monitoring a large part of the sky in spectrometer mode across the $10-88 \mathrm{MHz}$ bandwidth, bright transients can be identified in the spectral domain. Simultaneous detections in at least two stations will increase the reliability of any unanticipated transients. When seasonally passing through the beam, anticipated sources include Jupiter and the Sun. Depending on the latitude of a given station (now expanded to higher latitudes with the addition of Station V in Michigan), each station is capable of daily monitoring of nearby radio pulsars within the appropriate declination range. Rare, giant pulses may be detectable above the background by identifying their unique dispersion measures with the spectrometer. We also expect a strong sensitivity to broadband ionospheric activity.

\section{DESIGN}

LoFASM stations are built with 12 LWA antennas [1] per station in a two-ring structure [2], with the thirteenth antenna at each station functioning as an outrigger for the purpose of sky calibration. The six antennas of each concentric ring are combined in phase into one RF signal, for each of the two polarizations the cross-dipole LWA antennas provide. Each ring is analog-phased such that the resulting time delay between the two rings should be zero for radio sources at the zenith. The correlated signal between the two rings then selects radio sources from the zenith-pointing drift scan beam; the anticorrelated signal between the two beams represents the horizon signal, more likely to be radio frequency interference
(RFI). The outrigger is placed $150 \mathrm{~m}$ to the west to enable interferometry between it and the main rings; mapping known bright point sources can calibrate the instrument in Jy.

The six input RF signals (two rings and one outrigger, two polarizations each) are transmitted to the control shed about $70 \mathrm{~m}$ away by buried coaxial cables. At the shed, the cables pass through a bias tee, enabling a DC current that powers the front-end electronics boards on top of the LWA antennas. Next in the signal chain, the RF signals pass through an analog receiver, finally passing into the ADCs of the backend receiver, a Reconfigurable Open Architecture Computing Hardware (ROACH) board from the Berkeley CASPER group, with appropriate clocking to digitize the signal. Within the ROACH board the digitized voltage time series is Fourier transformed into the spectral domain using a Xilinx Field Programmable Gate Array (FPGA). The resulting dynamic spectra, with a $80 \mathrm{~ms}$ time resolution, are stored to disk on the control computer linked to the ROACH board. The time resolution can be improved in a future implementation, but we preferred a straightforward setup for the initial test of the instrument.

All steps of the construction of Station V involved Hillsdale College students, who developed software, hardware, and electrical engineering skills. The straightforward and smallscale design of each station is intended for replication at other small, undergraduate-focused institutions, with enormous pedagogical value. Additionally, the small stations do not need to be in ultra radio-quiet areas, because a significant fraction of local RFI can be identified as such both through the horizon rejection method and the astrophysical requirement for detection at multiple, very distant stations.

\section{ATtAining FiRst Light}

In order to verify the telescope's operation, we searched for the diurnal cycle of the Milky Way's low-frequency radio emission passing through the beam twice per day in data from a single ring. A daily cycle with two peaks was indeed found at the expected LSTs on repeated days. Next, switching to interferometer mode, we used the inner ring EW polarization with the outrigger EW polarization and constructed the crosspower between the two, in a single spectrogram spanning the day showing fringes. We then took the delay spectrum, which featured two S-shaped curves corresponding to the expected daily transits of Cygnus A and Cassiopeia A. Five-minute radar sweeps were occasionally but repeatedly seen at the low 
end of the band, showing that the telescope was operational and sensitive to non-local RFI.

\section{A Detailed Data Stream}

After weeks of operation, several interesting events were recorded shortly after midnight on separate days, such as the one shown in Figs. 1 and 2 from June 15th, 2019. Lasting about $40 \mathrm{~min}$, the event clearly has a strong RFl component, with a narrowband feature around $55.6 \mathrm{MHz}$, and a higher frequency cutoff. The broadband features across the $5 \mathrm{MHz}$ span of the activity includes complex looping and crisscross features, at least qualitatively reminiscent ([3], [4]) of ionospheric events such as traveling ionospheric disturbances (TIDs), in this case possibly interacting with narrowband transmissions. Two other shorter events of 1-5 min were also seen shortly after midnight on other days, but apart from these three events, most observed days in June showed no similar activity. (The two shorter events also featured a bright narrowband feature at the bottom of the frequency range, differing by a few $\mathrm{MHz}$ from 55.6 MHz.) The event in Figs. 1 and 2 nearly overlapped with the largest solar Kp-index of the month ${ }^{1}$. While we do not claim any particular ionospheric explanation for these data, they at least show the ability of LoFASM to detect unexpected, bright, broadband events with detailed spectral information.

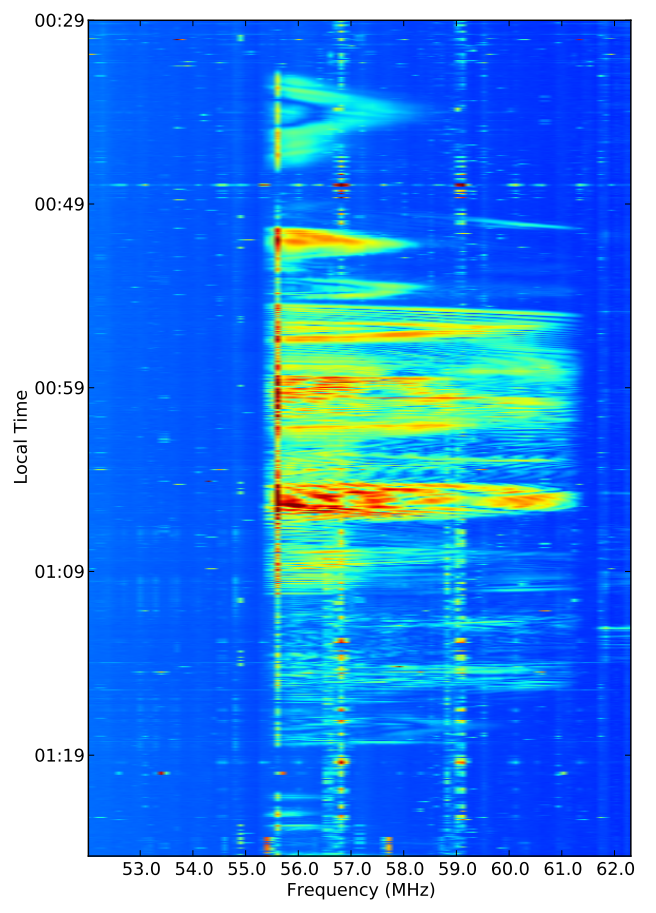

Fig. 1. Possibly ionospheric events detected with LoFASM Station V in Michigan on June 15, 2019. Color scaling is logarithmic in arbitrary units.

\footnotetext{
${ }^{1}$ https://www.gfz-potsdam.de/en/kp-index/
}

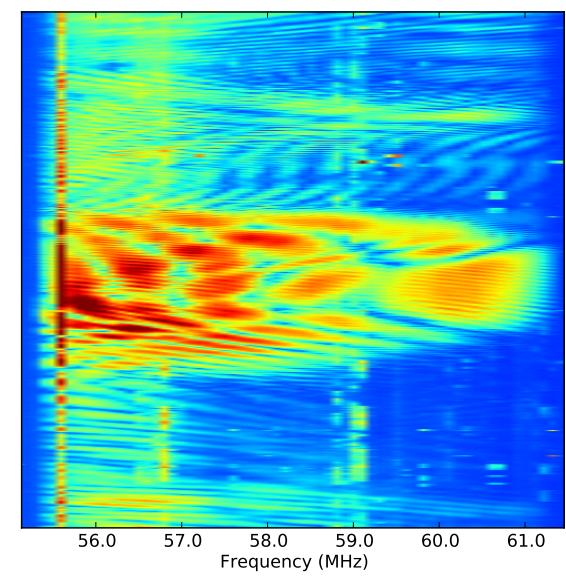

Fig. 2. Magnification of region in Fig. 1 dynamic spectrum near 01:05.

\section{NeXt Steps}

The LoFASM stations, having now all passed first light, can being to operate together as a network of largely overlapping drift-scan telescopes. We will continue to search for solar events, atmospheric events, and bright astrophysical transients such as giant pulses. In the coming years, LoFASM infrastructure and resources may coordinate with the proposed LWASwarm telescope [5], a series of mini-stations of 50 or more LWA antennas with small, undergraduate serving institutions taking the lead in maintaining their own local arrays.

\section{ACKNOWLEDGEMENT}

The Low-Frequency All-Sky Monitor V was funded by a Hillsdale College startup grant. Student research with LoFASM was funded by Hillsdale College's LAUREATES program. We are grateful to Xilinx, Inc., for the donation of an FPGA for educational use. We thank Murat Torlak at UT Dallas, for valuable input about constructing a source map.

\section{REFERENCES}

[1] Hicks, B.C., Paravastu-Dalal, N., Stewart, K.P., Erickson, W.C., Ray, P.S., Kassim, N.E., Burns, S., Clarke, T., Schmitt, H., Craig, J., Hartman, J., and Weiler, K.W.: "A Wide-Band, Active Antenna System for Long Wavelength Radio Astronomy" (2012), Publications of the Astronomical Society of the Pacific 124, 1090.

[2] Dartez, L. P.: "A Digital Backend for the Low Frequency All Sky Monitor." (2014) Masters Thesis.

[3] Koval, A., Chen, Y., Stanislavsky, A., and Zhang, Q.-H.: "Traveling ionospheric disturbances as huge natural lenses: Solar radio emission focusing effect" (2017) Journal of Geophysical Research (Space Physics) 122, 9092.

[4] Koval, A., Chen, Y., Tsugawa, T., Otsuka, Y., Shinbori, A., Nishioka, M., Brazhenko, A., Stanislavsky, A., Konovalenko, A., Zhang, Q.-H., Monstein, C., and Gorgutsa, R.: "Direct Observations of Traveling Ionospheric Disturbances as Focusers of Solar Radiation: Spectral Caustics" (2019) The Astrophysical Journal 877, 98.

[5] Taylor, G., Dowell, J., Pihlström, Y., Schinzel, F., Kassim, N., Hallinan, G., Hoffman, I.M., Besson, D., Prohira, S., Gretarsson, A.M., Fobes, R.D., Maccarone, T.J., Dolch, T., Bowman, J .D., Jacobs, D. C., Jenet, F. A., Kurtz, S., Thyagarajan, N., Beardsley, A. P., Burns, J. O., Bullett, T., Kavic, M., and Driessen, P. "The Swarm Development Concept for the LWA" (2019) Bulletin of the American Astronomical Society 51, 2. 